\section{New Web Location for Annual and Weekly NNDSS Data}

To improve the usability, availability, quality, and timeliness of surveillance data $(I)$ as part of the CDC Surveillance Strategy, CDC will provide users with a convenient way to access notifiable infectious and noninfectious disease data through the National Notifiable Diseases Surveillance System (NNDSS) website.

CDC has redesigned the data and statistics section of the NNDSS website to be a one-stop shop for users to find both detailed information about notifiable disease data and links to the annual and weekly data. Although these data will no longer be published in their current format in $M M W R$, users can easily access the information through the NNDSS website. To ease the transition, $M M W R$ also will link users from its website to the new location on the NNDSS website.

\section{Annual Reporting}

CDC expects to transition the reporting of NNDSS annual data in November 2017. The redesigned NNDSS Data and Statistics webpage at https://wwwn.cdc.gov/nndss/data-andstatistics.html will contain links to infectious disease data tables that are available in HTML, text, and PDF formats and hosted on the CDC WONDER (2) platform. The webpage also will provide links to noninfectious condition and disease outbreak surveillance reports published by CDC programs and hosted on CDC WONDER. In addition, the webpage will provide the following resources: 1) documentation for NNDSS infectious diseases and noninfectious conditions and disease outbreaks, including how the data are collected, reported, and finalized; 2) publication criteria; 3) notes about interpreting data; and 4) the list of notifiable conditions by year.

\section{Weekly Reporting}

CDC expects to transition the reporting of NNDSS weekly data in January 2018 and will provide more information later this year.

Consolidating the notifiable disease data on the NNDSS website is part of the NNDSS Modernization Initiative (NMI) strategy to streamline NNDSS and access to data for users; NMI is a component of the CDC Surveillance Strategy. This consolidation of information also is in response to the recommendations of a workgroup consisting of representatives from the CDC Excellence in Science Committee, the Surveillance Science Advisory Group, and $M M W R$, to make more data available online and to allow $M M W R$ to focus on publishing scientific and actionable surveillance reports.

\section{Reference}

1. Richards CL, Iademarco MF, Anderson TC. A new strategy for public health surveillance at CDC: improving national surveillance activities and outcomes. Public Health Rep 2014;129:472-6. https://doi. org/10.1177/003335491412900603

2. CDC. CDC WONDER. Atlanta, GA: US Department of Health and Human Services, CDC; 2017. https://wonder.cdc.gov/ 


\section{Vol. 66 No. 34}

In the report, "Notes from the Field: Lead Poisoning in an Infant Associated with a Metal Bracelet - Connecticut, 2016" on page 916, the second paragraph should have read "The parents reported that the child intermittently wore a handmade "homeopathic magnetic hematite healing bracelet" that they had purchased from an artisan at a local fair (Figure). The bracelet was described as "homeopathic," but homeopathic products are, by definition, regulated drugs and so nondrug items, such as bracelets, cannot be homeopathic. Cases of mislabeled products, especially among homemade items, should raise suspicion for consumers and health care professionals. The child wore the bracelet for teething related discomfort and was sometimes noted to chew on it. Small spacer beads from the bracelet tested at the Manchester Health Department were positive for lead (17,000 ppm). No identifying marks indicating metal content or manufacturer were found on the bead. The vendor records were not available, and the bracelet maker could not be located."

\section{Vol. 66 No. 34}

In the report, "Overdose Deaths Related to Fentanyl and Its Analogs — Ohio, January-February 2017,” on page 904, the sixth sentence of the first paragraph should have read "The Wright State University and the Montgomery County Coroner's Office/Miami Valley Regional Crime Laboratory (MCCO/MVRCL) collaborated on a National Institutes of Health study of fentanyl analogs and metabolites and other drugs identified in 281 unintentional overdose fatalities in $\mathbf{2 5}$ Ohio counties during January-February 2017."

On page 904 the second sentence of the second paragraph should have read "Data from 281 unintentional overdose fatalities that occurred in Montgomery County and $\mathbf{2 4}$ additional counties $^{\dagger}$ during January and February 2017, were analyzed by the MCCO Toxicology laboratory, and had assigned causes of death as of May 8, 2017, were included in this study."

On page 905, under "What is added by this report?" the first sentence should have read "Approximately $90 \%$ of unintentional overdose deaths examined in $\mathbf{2 5}$ Ohio counties that occurred during January-February 2017 involved fentanyl, fentanyl analogs, or both, whereas heroin was identified in the minority $(6 \%)$ of cases, with somewhat higher prevalence in Appalachian counties."

On page 907, the second sentence of the first paragraph should have read "Approximately $90 \%$ of unintentional overdose deaths in 25 Ohio counties that occurred during January and February 2017 involved fentanyl, fentanyl analogs, or both.

On page 907, the last sentence of the third paragraph should have read "Finally, data were obtained from 25 Ohio counties, and findings might not be generalizable to the entire state." 